\title{
CAPSOCA: Hybrid technique for nosologic segmentation of primary brain tumors
}

\author{
Shafaf Ibrahim ${ }^{1}$, Noor Elaiza Abd Khalid ${ }^{2}$, Mazani Manaf ${ }^{3}$ \\ ${ }^{1}$ Faculty of Computer and Mathematical Sciences, Universiti Teknologi MARA (Melaka), Malaysia \\ ${ }^{2,3}$ Faculty of Computer and Mathematical Sciences, Universiti Teknologi MARA (Shah Alam), Selangor, Malaysia
}

\begin{tabular}{l}
\hline \hline Article Info \\
\hline Article history: \\
Received Jan 25, 2019 \\
Revised Feb 20, 2019 \\
Accepted May 15, 2019 \\
\hline
\end{tabular}

\section{Keywords:}

Clustering algorithm

(CAPSOCA)

Grey level co- occurrence

matrices (GLCM)

Intensity based analysis

Magnetic resonance imaging

(MRI)

Particle swarm optimization (PSO)

\begin{abstract}
Detection of primary brain tumors is inspired by the necessity of high accuracy as it deals with human life. Various imaging modalities techniques have incarnated as a tool in diagnosis and treatment domain. Yet, experienced and competent medical practitioners for the proper interpretation are still required. Thus, the involvement of information technology is highly demanded in introducing reliable and accurate computer systems. This study presents an algorithm for nosologic segmentation of primary brain tumors on Magnetic Resonance Imaging (MRI) brain images. Nosologic refers to the classification of diseases that can facilitate the diagnosis of neurological diseases. The purpose of segmentation is to highlight the tumor areas, whereas classification is used to identify the type of the primary brain tumors. For this purpose, an algorithm which hybridized the Grey Level Co- occurrence Matrices (GLCM), Intensity Based Analysis (IBA), Adaptive Network-based Fuzzy Inference System (ANFIS) and Particle Swarm Optimization (PSO) Clustering Algorithm (CAPSOCA) is proposed. The combination of several computer vision techniques is aim to deliver reproducible nosologic segmentation of primary brain tumors which are gliomas and meningiomas. The performance of the CAPSOCA is quantified by two measurements which are segmentation and classification accuracy. The segmentation accuracy is evaluated using comparison with ground truth approach. On the other hand, the classification accuracy is quantified using a truth table by comparing the classification outcomes with histopathology diagnosis. Upon the testing conducted, the CAPSOCA was proven to be an effective algorithm for nosologic segmentation of primary brain tumors. It appeared to return $88.09 \%$ of overall mean accuracy for gliomas segmentation, $86.92 \%$ of overall mean accuracy for meningiomas segmentation. In another note, $83.72 \%$ and $85.19 \%$ of classification accuracy for gliomas and meningiomas were observed.
\end{abstract}

Copyright (C) 2019 Institute of Advanced Engineering and Science. All rights reserved.

\footnotetext{
Corresponding Author:

Shafaf Ibrahim,

Faculty of Computer and Mathematical Sciences,

Universiti Teknologi MARA (Melaka),

77300 Merlimau, Melaka, Malaysia.

Email: shafaf2429@uitm.edu.my
}

\section{INTRODUCTION}

A rapid development of medical imaging modalities such as X-ray, Computed Tomography (CT) scan, Ultrasound and Magnetic Resonance Imaging (MRI) has enabled the investigations of domains that encompass areas that were out of reaches for the human's eyes. These modalities make it possible to explain the structures of organs and cells, enable the observation of the way they function, allows abnormalities or dysfunction detection as well as assisting in pathology diagnosis [1]. 
The brain is defined as one of the most complicated, least accessible and prone to complex abnormalities in human organs [2]. Accordingly, medical imaging modalities are anticipated to contribute the primary benefits in overcoming these circumstances. The brain complexity can be expressed at a variety of scales. Therefore, a deeper understanding of the brain anatomical structures plays a crucial role in the search for more efficient brain lesions and diseases (brain tumor, infarction, haemorrhage, stroke and many other brain abnormalities). Detection of tumors in brain tissue area from different medical images is inspired by the necessity of high accuracy as it deals with human life [3]. Presently, various imaging modalities techniques have incarnated as a tool for the doctors and radiologists to help them in diagnosis and treatment domain. While these are highly accurate and fast, they still require experienced and competent medical practitioners for the proper interpretation [4]. Thus, computer aided diagnosis is significantly useful due to the fact that it could enhance the results of humans in such domain $[5,6]$.

The involvements of information technology in the development of various applications have completely changed the world. The noticeable reasons for the introduction of computer systems are reliability, accuracy, simplicity and ease of use [5-7]. Moreover, it has become almost compulsory to use computers to assist radiological experts in clinical diagnosis and treatment planning due to the increasing use of imaging for diagnosis, treatment planning and clinical studies [8,9]. In the past several years, computer aided system has widely applied for many ranges of application such as a brain tumour [10], lung cancer [11] and breast cancer [12]. Computer vision is a branch of Artificial Intelligence (AI) that provides computers with the functions characteristic of human vision. Currently, computer vision has produced important applications in image processing perspective such as segmentation [13-15], feature extraction [16], texture classification [17] and many other applications.

PSO has been observed to be successfully applied for clustering approach in many research and application areas, as well as various representations of methods for image segmentation. It has also gained increasing popularity in recent years due to its ability to solve efficiently and effectively an increasing number of problems in science and engineering as in these numerous studies [18-20]. It is reported that the PSO outperformed other methods such as Fuzzy c-Means (FCM) [21], K-means, Genetic Algorithm (GA) and Differential Evolution (DE) [22], and even the other Swarm Intelligence (SI) algorithms which are Ant Colony Optimization (ACO), Artificial Bee Colony Algorithm (ABCO) and Bacterial Foraging Algorithm (BFOA) [20]. Therefore, this paper presents a hybrid algorithm known as CAPSOCA for nosologic segmentation of primary brain tumors on Magnetic Resonance Imaging (MRI) brain images. The algorithm is designed as a PSO-based clustering algorithm which combines several computer vision techniques which are GLCM, IBA, and ANFIS. The GLCM and IBA acted as feature extraction techniques in determining the patterns and characteristics for the various types of brain tissues. The basic concept is that the local textures in the images can reveal the biological structures of gliomas and meningiomas. Alternatively, the ANFIS is implemented to classify the type of primary brain tumors. The encapsulation of all the implemented techniques into the PSObased clustering algorithm produced a hybrid technique called as CAPSOCA. The organization of the rest of this paper is as follows: Section 2 presents our proposed research method, including the brief description on data collection, and the CAPSOCA structure. Section 3 presents our results and discussions. Finally, we present our conclusions in Section 4.

\section{RESEARCH METHOD}

The data collection involved 167 Fluid Attenuated Inversion Recovery (FLAIR)-MRI brain images of gliomas and menigiomas which were collected from Hospital Sungai Buloh, Selangor. The aim of this study is to generate an innovative non-invasive method for nosologic segmentation of primary brain tumors in the MRI field. The concept is mainly focused on the development of a comprehensive algorithm which combines several computer vision techniques for nosologic segmentation of primary brain tumors which are gliomas and meningiomas. The basic concepts of the PSO, the proposed hybrid technique of CAPSOCA, including the description of all techniques involved are explained in the next subsection.

\subsection{The PSO}

PSO is an evolutionary computation technique proposed by Kennedy and Eberhart in 1995. The basic idea of PSO is inspired by social behaviour of bird flocking, fish schooling and swarm theory. It is a SI based algorithms that have been applied in numerical optimization problems [23]. PSO is a very popular SI algorithm for global optimization over continuous search spaces. It has attracted the attention of several researchers which resulting in a huge number of variants of the basic algorithm as well as many parameter automation strategies ever since its advent in 1995 In the past several years, PSO has been successfully applied in many research and application areas. One of the advantages of PSO is that it is easier to implement and there are very few parameters to adjust [24]. It has also demonstrated as a faster and cheaper way of obtaining better results as 
compared with other methods [25]. Reference [26] proposed a particle swarm based segmentation algorithm for automatically grouping the pixels of an image into different homogeneous regions. They used PSO for the fuzzy segmentation of images since the pixels in an image generally have high correlation and have proven that PSO performed well.

In implementing the PSO, several matters should first be considered in order to apply the PSO idea such as the representation of initial population, representation of position and velocity strategies, fitness function identification and the limitation. PSO is defined by considering five essential parameters as tabulated in Table 1.

Table 1. PSO parameters

\begin{tabular}{cl}
\hline Parameters & \multicolumn{1}{c}{ Description } \\
\hline Particle & candidate solution to a problem \\
Velocity & the rate of position change \\
Fitness & the best solution achieved \\
pcurrent & a position value of the current particle \\
pbest & present best, the best value obtained in previous particle \\
gbest & global best, the best value obtained so far by any particle in the population \\
\hline
\end{tabular}

\subsection{The CAPSOCA}

The CAPSOCA is proposed in which several computer vision techniques are combined throughout the PSO-based algorithm. The combination of GLCM and IBA is used to analyze the texture of the brain component categories. Whereas, the ANFIS plays the texture classification roles which classify the texture to the category it belongs to. Finally is the implementation of PSO algorithm which acted as the search and optimization method in finding the best pixels for segmentation, and also as the outline for the whole nosologic segmentation algorithm. The pictorial representation of the proposed nosologic CAPSOCA segmentation model is illustrated in Figure 1.

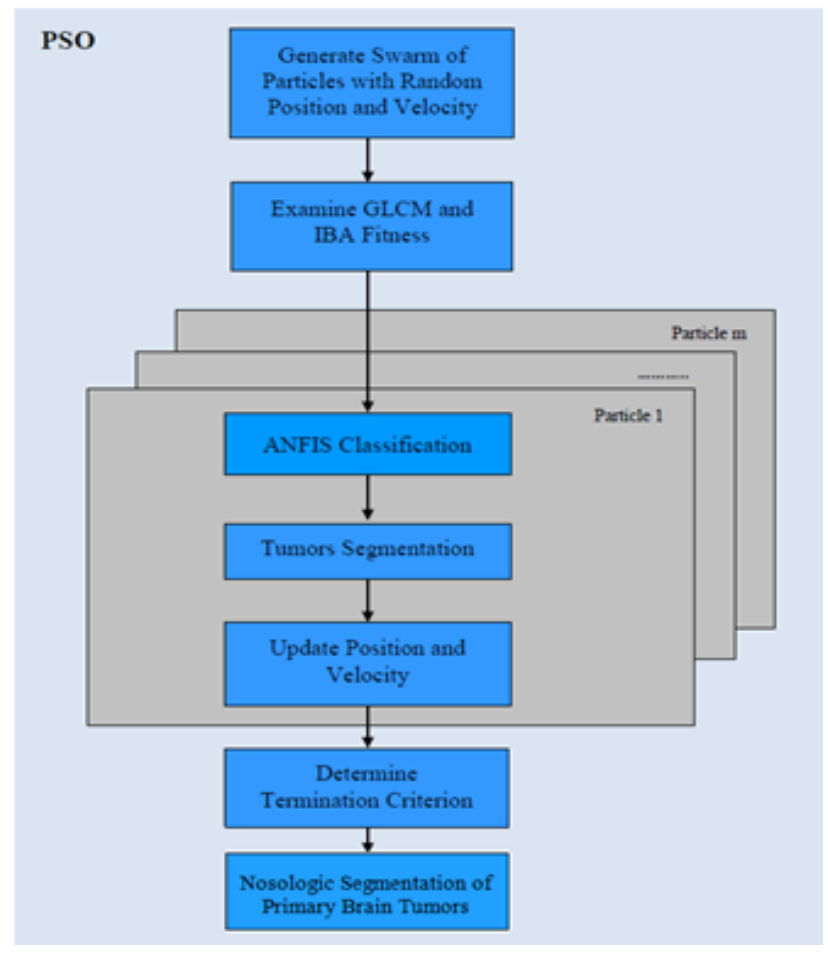

Figure 1. Proposed nosologic capsoca segmentation model

The six primary steps of CAPSOCA segmentation are elaborated as below:

a. Step 1: Generation of Particles

PSO is initialized with a swarm of random solutions called particles. Each particle maintains its own position, velocity and its current best position. The algorithm is initialized to contain 500 points of particles 
with random position and velocity. The points had been randomly selected in the $\mathrm{X}$-axis value within the image width while the Y-axis value within the image height. Therefore, the computer will automatically generate 500 points from the image randomly without considering the position of the points.

b. Step 2: GLCM and IBA Fitness Examination

After the swarm of particles is generated, each particle's fitness needs to be examined based on the Region of Interest (ROI) table which acted as the objective function. An objective function refers to a particular type of function that quantifies the solution of an optimization problem. This function is specific to each optimization problem and its suitability is essential to contribute a success and performance of the particular optimization process. An ideal objective function correlates closely with the goal of a particular algorithm. In some cases, it is very hard or impossible to come up even with a guess of what fitness function definition might be. In this study, the ROI table combines both GLCM and IBA parameters. The pattern and characteristics of each particle and its $3 \times 3$ matrix surrounding pixels are examined according to the parameters values defined in the reference table. The objective function used is formulated as in (1):

$$
\begin{aligned}
& \text { Min }\left(\text { ObjFunc }_{p c}\right) \\
& \text { Max }\left(\text { ObjFunc }_{p c}\right) \\
& \text { Mean }\left(\text { ObjFunc }_{p c}\right)
\end{aligned}
$$

where $\mathrm{p}=0,1,2,3,4,5,6$ and $\mathrm{c}=0,1,2,3$. $\mathrm{p}$ represents the seven parameters of GLCM and IBA which are energy, entropy, contrast, dissimilarity, min, max and mean. Whereas, the c represents the four brain component categories of dark gliomas, light gliomas, dark meningiomas and light meningiomas.

\begin{tabular}{|c|c|c|}
\hline Method & Parameter & Description \\
\hline & Energy & Provides the sum of squared elements in the GLCM \\
\hline & Entropy & Measures the uncertainty or disorder \\
\hline & Contrast & Measures the local variations in the GLCM \\
\hline GLCM & Dissimilarity & $\begin{array}{l}\text { Measures the discrepancy between the two objects based on several } \\
\text { features. Dissimilarity may also be viewed as a measure of disorder } \\
\text { between two objects }\end{array}$ \\
\hline \multirow{3}{*}{ IBA } & Min & Minimum grey level pixel value occurred \\
\hline & $\operatorname{Max}$ & Maximum grey level pixel value occurred \\
\hline & Mean & Mean of grey level pixel value occurred \\
\hline
\end{tabular}
The parameters are further described as in Table 2.

Table 2. Parameters of reference table

These values are then matched with the same parameters of the current particle so that the current particle could be classified into the particular brain component it belongs to. The classification of each particle is performed using ANFIS technique.

c. Step 3: ANFIS Classification

The classification of each fit particle is achieved using a classification method of ANFIS. The step by step for each layer of ANFIS classification is presented in a flow chart as shown in Figure 2. After a particular fit particle is classified by the ANFIS, the particle is then segmented according to the brain component categories it belongs to. Each region of tumors is assigned to different colors with the purpose that the segmentation of each region is noticeable.

d. Tumor Segmentation

Segmentation of medical images holds an important position in the area of image processing. It is used to extract information from complex medical images and it has wide application in the medical field. The main objective of tumors segmentation in this study is to segment the region of tumors which are gliomas and meningiomas. Table 3 tabulates a sample of CAPSOCA segmentation for gliomas and meningiomas.

e. Step 5: Position and Velocity Update

Next, each current pbest particle is evaluated in the same way as the particles generated initially. Each best fitted particle among all the particles is stored as global best (gbest). This process is done iteratively which the fitness's, position and velocity are updated in every iteration if necessary. The iteration of update rules of position and velocity leads to the exploration of the whole regions that turn out to be the final outcomes. Velocity refers to the rate of position change which the particle's position is depending on the velocity. In the first iteration, four neighbouring pixels of each particular pbest particle are considered. There are four coordinates of neighbouring pixels considered are $(x, y-1),(x-1, y),(x, y+1)$ and $(x+1, y)$.

Indonesian J Elec Eng \& Comp Sci, Vol. 16, No. 1, October 2019 : 267 - 274 


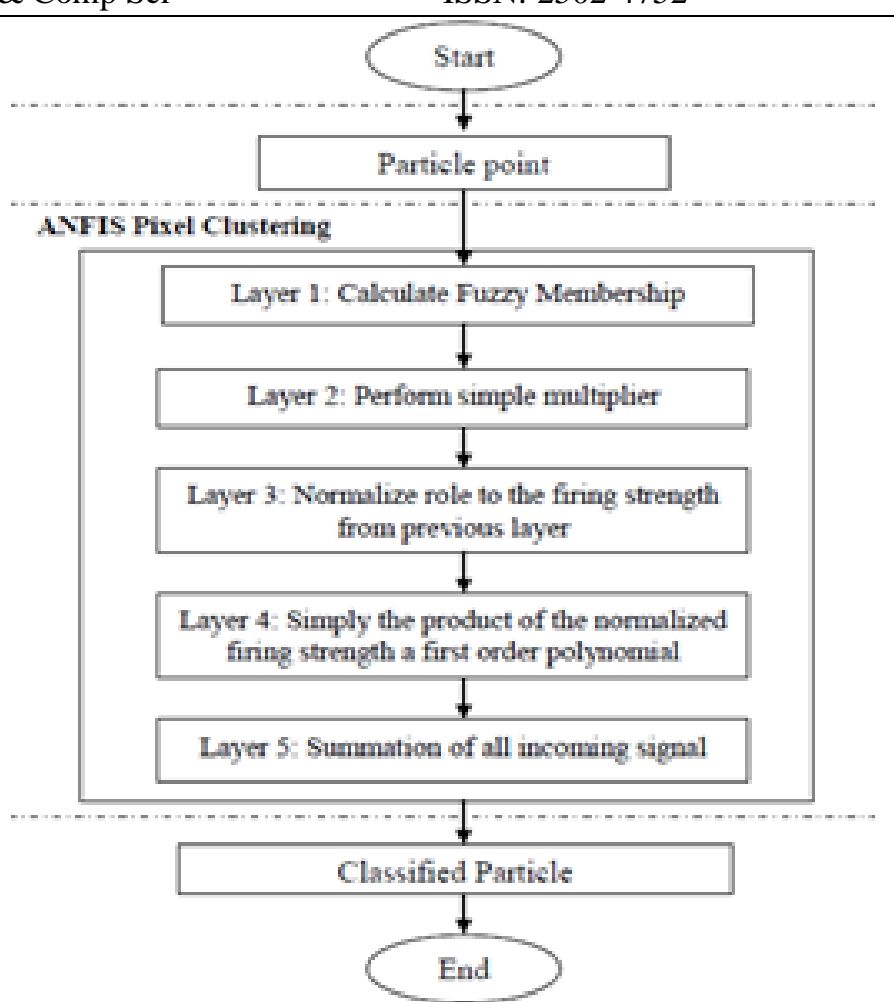

Figure 2. Flowchart of ANFIS classification

Table 3. Samples of CAPSOCA segmentation

Category




\section{f. Step 6: Determine Termination Criteria:}

The processes of fitness examination, velocity and position update are performed constantly until the termination criteria are met. These criteria are determined based on the problem to be optimized. Therefore, the process will halt and return the result when there are no unprocessed pbest and gbest pixels in the maximum velocity regions. Otherwise, the process will continue to start the fitness examination, velocity and position updating processes for the next ith particles. The maximum velocity is used to keep the particles from moving too far beyond the search space. The maximum velocity in this study is limited to the whole region of each MRI brain image. It is chosen for facilitating global exploration of particle's position since too low maximum velocity region might lead to the difficulties of particles in exploring the optimal regions.

\subsection{Evaluation of Accuracy}

The CAPSOCA segmentation quality and the level of accuracy for gliomas and meningiomas are quantified using statistical values which are the mean percentage of sensitivity, specificity and accuracy as in (2)-(4).

$$
\begin{aligned}
& \text { Sensitivity }=\frac{\text { True Positive }}{\text { True Positive }+ \text { False Negative }} \times 100 \% \\
& \text { Specificity }=\frac{\text { True Negative }}{\text { True Negative }+ \text { False Positive }} \times 100 \% \\
& \text { Accuracy }=\frac{\text { True Positive }+ \text { True Negative }}{\text { True Positive }+ \text { True Negative }+ \text { False Positive }+ \text { False Negative }} \times 100 \%
\end{aligned}
$$

On the other hand, the primary brain tumors classification accuracy is evaluated using a truth table. A truth table is a useful assessing method for determining the validity or invalidity of any form of argument. Pixel-based diagnosis is proposed to classify the types of primary brain tumors based on the tumor- segmented pixels. It is done by comparing the percentage values of pixel-based diagnosis for both gliomas and meningiomas. The percentage value of pixel-based is calculated by the numbers of tumor-segmented pixels which overlapped with the ground truth. The pixel-based diagnosis is determined according to the highest percentage of overlapped pixel value between both types of primary tumors. Table 4 tabulates a number of samples on how the pixel-based diagnosis is identified.

Table 4. Samples of pixel-based diagnosis

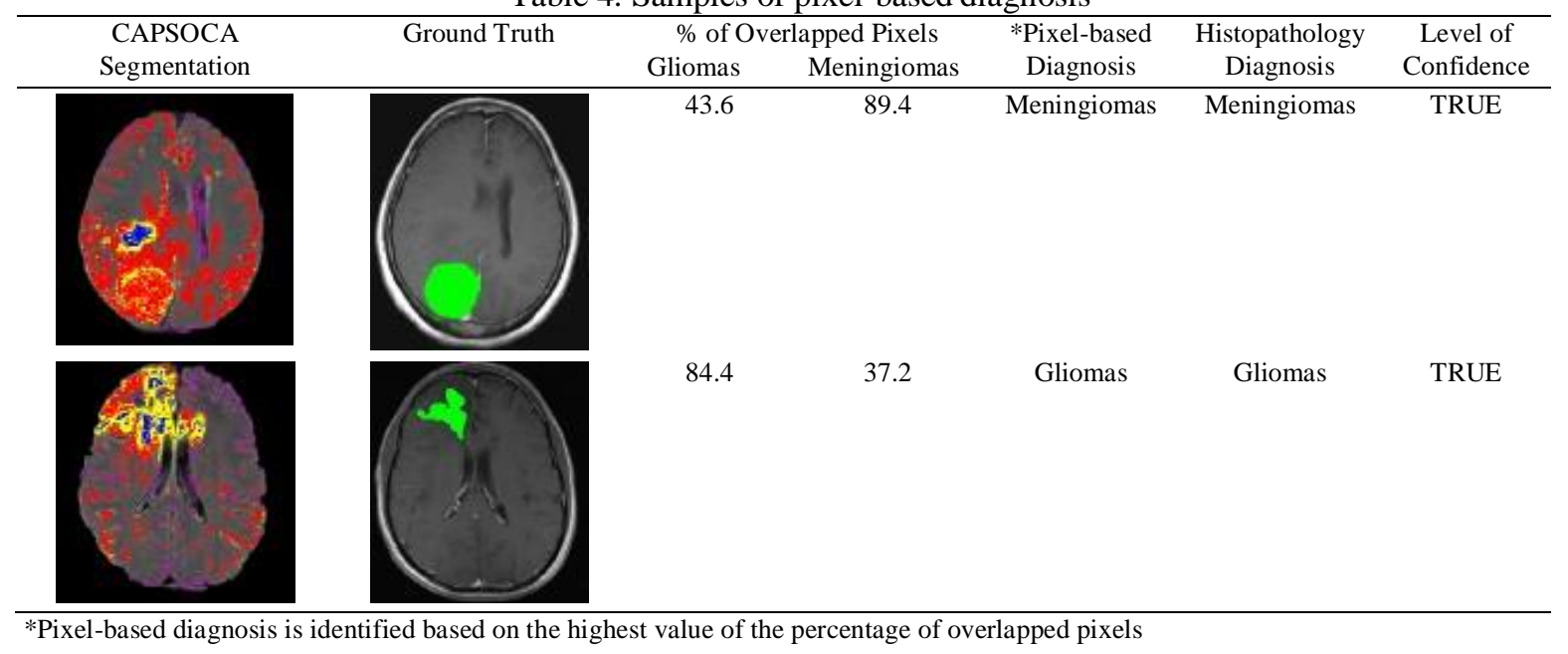

Next, the level of confidence measured the accuracy of the pixel-based diagnosis by comparing the outcomes with histopathology diagnosis collected from the patient's record. The level of confidence is divided into two conditions which are TRUE or FALSE. Consequently, the percentage of accuracy for the pixel-based diagnosis model is calculated according to the number of TRUE pixel-based diagnosis to the total number of images as in (5).

$$
\% \text { of Classification Accuracy }=\frac{\text { No.of TRUE Pixel-based Diagnosis }}{\text { No.of images }}
$$




\section{RESULTS AND ANALYSIS}

The performance of the CAPSOCA is quantified by two measurements which are segmentation accuracy and classification accuracy. The segmentation accuracy is evaluated using comparison with ground truth approach. Table 5 tabulates the CAPSOCA segmentation summary for both gliomas and meningiomas.

Table 5. Summary of gliomas and meningiomas segmentation

\begin{tabular}{|c|c|c|c|}
\hline \multirow[t]{2}{*}{ Category } & \multicolumn{3}{|c|}{ Basic Quantifier } \\
\hline & Sensitivity $\%$ & Specificity \% & Accuracy $\%$ \\
\hline Gliomas & 78.55 & 88.62 & 88.09 \\
\hline Meningiomas & 53.71 & 88.34 & 85.92 \\
\hline
\end{tabular}

As seen from the Table 5, the CAPSOCA is observed to produce good segmentation results for gliomas. The statistic shows that the sensitivity, specificity and accuracy mean values produced consistent outcomes which are $78.55 \%, 88.62 \%$, and $88.09 \%$ respectively. These proved that the CAPSOCA is capable of producing good quality of segmentation in gliomas.

On the other hand, the CAPSOCA segmentation outcomes for meningiomas are also cannot be underestimated since it produced high mean values of specificity and accuracy, which are $88.34 \%$ and $85.92 \%$. However, a slightly low mean value of sensitivity is observed. This makes the segmentation of meningiomas is not as good as gliomas. The mean of sensitivity value proved that a high rate of False Negative might occur in segmentation which the CAPSOCA is incapable to segment the area it supposed to be. This is caused by confusion in distinguishing the texture homogeneity between the meningiomas with other anatomical brain structure such as the membrane area.

On the other hand, the classification accuracy is quantified using a truth table by comparing the classification outcomes with histopathology diagnosis collected from the patient's record. Table 6 summarizes the truth table results for gliomas and meningiomas classification.

Table 6. Summary of gliomas and meningiomas classification

\begin{tabular}{ccccc}
\hline Category & $\begin{array}{c}\text { No. of } \\
\text { Images }\end{array}$ & $\begin{array}{c}\text { No. of TRUE } \\
\text { Classification }\end{array}$ & $\begin{array}{c}\text { No. of FALSE } \\
\text { Classification }\end{array}$ & $\begin{array}{c}\% \text { of } \\
\text { Accuracy }\end{array}$ \\
\hline Gliomas & 86 & 72 & 14 & 83.72 \\
Meningiomas & 81 & 69 & 12 & 85.19 \\
\hline
\end{tabular}

Referring to Table 6, the primary brain tumors classification accuracy is evaluated to 86 images of gliomas and 81 images of meningiomas. The percentage of accuracy for meningiomas is observed to produce higher percentage value of $85.19 \%$ as compared to the gliomas which returned a slightly lower value of $83.72 \%$ of accuracy. Based on the performance, it can be concluded that the CAPSOCA showed a great potential for primary brain tumors classification.

\section{CONCLUSION}

This paper presents a CAPSOCA algorithm for nosologic segmentation of primary brain tumors on Magnetic Resonance Imaging (MRI) brain images. The application to 167 Fluid Attenuated Inversion Recovery (FLAIR)-MRI brain images has been successful. The performance of the CAPSOCA towards the gliomas and meningiomas had been evaluated by two measurements which are segmentation accuracy and classification accuracy. The CAPSOCA is observed to produce good segmentation results for gliomas as compared to the meningiomas. The performance obtained exhibit a little variation in segmenting the meningiomas due to False Negative value which affect the mean of sensitivity value. In contrast, the CAPSOCA returned high classification accuracy for both gliomas and meningiomas. Therefore, it can be concluded that the proposed hybrid algorithm of CAPSOCA is found to be successful as it showed a great potential for nosologic segmentation of primary brain tumors. It is expected to offer a new way to produce high resolution nosologic segmentation of MRI brain images which may help medical practitioners in making noninvasive decision steps in the future.

\section{ACKNOWLEDGEMENTS}

The research was supported by Ministry of Education Malaysia (MoE), and Universiti Teknologi MARA through the Fundamental Research Grant Scheme (FRGS) (600-IRMI/FRGS 5/3 (215/2019)). 


\section{REFERENCES}

[1] T. W. Way, M. H. Lubomir, S. Berkman, C. Heang-Ping, N. C. Philip, A. K. Ella, B. Naama, and Z. Chuan, "Computer-aided diagnosis of pulmonary nodules on CT scans: Segmentation and classification using 3D active contours", Journal of Medical Physics, Vol. 33(7), pp. 2323-2337, 2006.

[2] A. Pitiot, J. Totman, and P. Gowland, "Null Point Imaging: A Joint Acquisition/Analysis Paradigm for MR Classification", Medical Image Computing and Computer-Assisted Intervention - MICCAI 2007, ISBN: 978-3540-75756-6, pp. 759-766

[3] R. Bhattacharjee and M. Chakraborty, "Brain tumor detection from MR images: Image processing, slicing and PCA based reconstruction", Sch. of Bio-Sci. \& Eng., Third International Conference on Emerging Applications of Information Technology (EAIT), Jadavpur University Kolkata, India, pp. 97-101, 2012.

[4] A. Kothari, "Detection and classification of brain cancer using artificial neural network in MRI images", World Journal of Science and Technology, 2012, 2(5), pp. 01-04.

[5] M. A. Balafar, A. R. Ramli, and S. Mashohor, "Edge-preserving Clustering Algorithms and Their Application for MRI Image Segmentation", Proceedings of the MultiConference of Engineers and Computer Scientists, 2010, Volume 1, IMECS 2010, Hong Kong.

[6] E. Pietka, J. Kawa, P. Badura, and D. Spinczyk, “Open architecture computer-aided diagnosis system”, The Journal of Knowledge Engineering, Volume 27(1), pp. 17-39, 2010

[7] Masroor M. A., Dzulkifli M., Segmentation of Brain MR Images for Tumour Extraction by Combining K- means Clustering and Perona-Malik Anisotropic Diffusion Model, International Journal of Image Processing, Vol. 2(1), 2008.

[8] N. Sharma and L. M. Aggarwal, "Automated medical image segmentation techniques", Journal of Medical Physics, Vol. 35(1), pp. 3-14, 2010.

[9] R. B. Dubey, M. Hanmandlu, and S. K. Gupta, "Semi-automatic Segmentation of MRI Brain Tumor". ICGSTGVIP Journal, ISSN: 1687-398X, August, Vol. 9(4), 2009.

[10] R. Logeswaran, "Computer Aided Medical Image Analysis for Intra-Operative Low-Field MRI in Neurosurgery", 2010, Unpublished article. Universiti Multimedia Malaysia.

[11] R. Sammouda, J. A. Hassan, M. Sammouda, A. Al-Zuhairy, and H. ElAbbas, "Computer Aided Diagnosis System for Early Detection of Lung Cancer Using Chest Computer Tomography Images”, GVIP 05 Conference, December 2005, CICC, Cairo, Egypt, 2005.

[12] D. Xian-Fen, Z. Xin-Yu, W. Tian-Fu, C. Si-Ping, Y. Ying, and Z. Ling, "Highly Sensitive Computer Aided Diagnosis System for Breast Tumor Based on Color Doppler Flow Images”, Journal of Medical Systems, 2010.

[13] T. X. Pham, P. Siarry, and H. Oulhadj, "Integrating fuzzy entropy clustering with an improved PSO for MRI brain image segmentation", Applied Soft Computing, vol. 65, pp. 230-242, 2018. ISSN 1568-4946.

[14] K. Huang, Z. Wu, H. Peng, M. Tsai, Y. Hung, and Y. Lu, "A memetic particle gravitation optimization algorithm for solving image segmentation," 2018 IEEE International Conference on Applied System Invention (ICASI), Chiba, pp. 82-85, 2018.

[15] S. Ibrahim, N. E. A. Khalid, M. Manaf, and U. K Ngah, "Particle swarm optimization vs seed-based region growing: Brain abnormalities segmentation”, International Journal of Artificial Intelligence, 174-188, 2011.

[16] A. F. H., Alharan, H. K. Fatlawi, N. S. Ali, "A cluster-based feature selection method for image texture classification”, Indonesian Journal of Electrical Engineering and Computer Science, June, Vol. 14(3), pp. 14331442, 2019.

[17] Kahya M. A., "Classification enhancement of breast cancer histopathological image using penalized logistic regression", Indonesian Journal of Electrical Engineering and Computer Science, Vol.13, No.1, January 2019, pp. 405-410.

[18] R. Chakraborty, R. Sushil, and M. L. Garg, "An Improved PSO-Based Multilevel Image Segmentation Technique Using Minimum Cross-Entropy Thresholding”, Arab J Sci Eng, pp. 1-16, 2018.

[19] N. Forghani, M. Forouzanfar, A. Eftekhari, S. M Moradi, and M. Tesnehlab, "Application of Particle Swarm Optimization in Accurate Segmentation of Brain MR Images”, InTech Education and Publishing, ISBN 978-9537619-48-0, 2009.

[20] M. C. J. Christ, S. Sivagowri, and P. G. Babu, "Segmentation of Brain Tumors using MetaHeuristic Algorithms", Open Journal of Communications and Software, May, Vol. 1(1), 2014.

[21] M. Omran, A. P. Engelbrecht, and A. Salman, "Particle Swarm Optimization Method for Image Clustering", International Journal of Pattern Recognition and Artificial Intelligence, 19(3), pp. 297-322, 2005.

[22] W. Xiaoqiong and E. Z. Yin, "Image segmentation algorithm based on dynamic particle swarm optimization and K-means clustering”, International Journal of Computers and Applications, 2018.

[23] J. Kennedy and R.C. Eberhart, "Swarm intelligence", Morgan Kaufmann Publishers Inc., 2001, San Francisco, CA.

[24] A. Kaur A, "An Overview of PSO- Based Approaches in Image Segmentation", International Journal of Engineering and Technology, Vol 2(8), pp. 1349-1357, 2012.

[25] D. Das, A. Abraham, A., "Spatial Information Based Image Segmentation using a Modified Swarm Optimization Algorithm", Proceedings of the Sixth International Conference on Intelligent Systems Design and Applications, Vol. 2, 2006.

[26] A. Abraham, S. Das, and A. Konar, "Kernel Based Automatic Clustering Using Modified Particle Swarm Optimization", Proceedings of the 9th Annual Conference on Genetic and Evolutionary Computation, 2007, London, England, 2-9, ISBN: 978-1-59593-697-4. 